\title{
UNIDADES ECOLÓGICAS PARA UNA GESTIÓN ECOSISTÉMICA EN EL DISTRITO CARTAGENA DE INDIAS (COLOMBIA)
}

\section{ECOLOGICAL UNITS FOR ECOSYSTEM MANAGEMENT IN THE DISTRICT OF CARTAGENA (COLOMBIA)}

\author{
Edilbert Torregroza ${ }^{1}$, Mauro Hernández $^{2}$, Diana Barraza ${ }^{3}$, Alberto Gómez ${ }^{4}$, Francisco Borja ${ }^{5}$
}

\begin{abstract}
${ }^{1}$ Master en Gestión y Conservación del Medio Natural, Grupo de Investigación CTS, Universidad de Cartagena, Cartagena, Colombia, e-mail: etorregrozaf@unicartagena.edu.co; ${ }^{2,3,4}$ Ing. Alimentos. Auxiliares de investigación, Universidad de Cartagena, e-mail: ingmaurohernandez@gmail.com; dianabrzarh@gmail.com; agomezj@unicartagena.edu.co; ${ }^{5}$ Doctor en Geografía física. Docente Investigador Universidad de Huelva (Huelva - España), e-mail: fborja@uhu.es
\end{abstract}

Rev. U.D.C.A Act. \& Div. Cient. 17(1): 205-215, Enero-Junio, 2014

\section{RESUMEN}

Partiendo del enfoque ecosistémico, el presente trabajo plantea una caracterización del Distrito de Cartagena de Indias concibiéndolo como un territorio compuesto por un conjunto de unidades homogéneas, definidas desde el punto de vista de su trama natural y antrópica (Unidades Ecológicas de Gestión). La regionalización ecológica del área de estudio, se lleva a cabo mediante la clasificación de sus ecosistemas a escala de ecosección y su posterior caracterización, según los usos del territorio y principales atributos socio-económicos. Este conjunto de datos, se trata, espacialmente, mediante la herramienta Quantum GIS, obteniéndose un primer diagnóstico ambiental del Distrito, basado en una cartografía compuesta por un total de 14 Unidades Ecológicas de Gestión.

Palabras clave: Territorio, ecosistemas, unidades de gestión.

\section{SUMMARY}

Starting from the "ecosystem approach", this paper presents a characterization of the District of Cartagena de Indias (Colombia) understanding the entiy as a territory composed of a set of homogeneous units defined from the point of view of natural and anthropic framework (Ecological Management Units). Ecological regionalization of the study area was performed by using the eco-section scale, and further characterization according to the use and socioeconomic attributes of the territory. The processing of the spatial data was carried out employing QGIS, with which a preliminary diagnose was obtained, based on cartographic maps of the territory organization and of the identification of 14 Ecological Management Units in the District of Cartagena.

Key words: Territory, ecosystems, management units.

\section{INTRODUCCIÓN}

El enfoque ecosistémico constituye una propuesta de gestión integral del territorio, basada en el manejo de los sistemas naturales (Mitchell, 1999), desde donde se contribuye, tanto a la conservación de la naturaleza como al bienestar humano, lo cual, superado en parte la dialéctica "conservación vs. desarrollo", vigente desde la década de los ochenta, se puede considerar como una misma cosa (Millennium Ecosystem Assessment, 2003; Folke, 2006). Conceptualmente, esta aproximación resalta el papel de las interacciones que unen a la sociedad con su entorno natural, intentando evitar así la tradicional separación y el punto de vista parcelado y sectorial con el que, habitualmente, se desenvuelven las ciencias sociales y naturales (Jianguo et al. 2007). Desde esta perspectiva, la gestión ambiental se centra, en principio, en el estudio y en el manejo de las aludidas relaciones de interdependencia, hasta conferirles un significado preeminente en el plano de la acción política, asumiendo que los seres humanos y su diversidad cultural son una parte inherente del sistema natural, entendido en el sentido más amplio de la expresión (UNEP, 2006). En términos operativos, esta línea de pensamiento procede poniendo, permanentemente, en valor el principio, según el cual, ninguna iniciativa de alcance ambiental se puede llevar a cabo desconociendo el papel ejercido por los seres humanos sobre el territorio o separándolos de la naturaleza, dado que la actividad antrópica, 
hoy por hoy, es el factor que más profundamente modula el funcionamiento y la evolución de los sistemas naturales (Grumbine, 1994; Sala et al. 2000). Existen así diferentes miradas acerca del manejo de los ecosistemas, que derivan en múltiples aproximaciones sobre el significado de "enfoque ecosistémico". De cualquier manera, la Gestión Basada en Ecosistemas reconoce que la gente y sus valores son parte de los sistemas que deben ser tenidos en cuenta durante una gestión que se precie de ser integral; sin embargo, el grado de incidencia de los valores sociales en el resultado de la gestión basada en ecosistemas sigue siendo un aspecto que despierta discusión entre los estudiosos del tema (Yaffee, 1999).

Considerado en estos términos, el enfoque ecosistémico plantea un importante reto a los investigadores del territorio, habida cuenta de que buena parte de sus aportaciones están bastante alejadas en lo substancial de una realidad que es necesariamente dual: natural y antrópica. De ahí la necesidad de, por una parte, llevar a cabo una revisión de las problemáticas relacionadas, específicamente, con la gestión del territorio, las cuales, requieren cada vez más urgentemente de un diálogo transdisciplinar de saberes complementarios (Nicolescu, 1999), tanto entre científicos y tomadores de decisiones como entre académicos y ciudadanía en general (Morin, 2001) y, por otra, de la puesta en marcha de nuevas iniciativas encaminadas a favorecer la implementación e institucionalización de procesos participativos y de cualesquiera otras fórmulas colaborativas.

\section{Regionalización Ecológica y Unidades Ecológicas de Ges-} tión: La teoría de los sistemas jerárquicos, se sustenta sobre la idea de que cada uno los componentes de un determinado sistema constituyen, a su vez y a una escala diferente, otros sistemas con dimensiones y con complejidad propias, que se anidan en aquél. Aplicado al medio natural, se puede entender que cada ecosistema, una vez dimensionado espacial y temporalmente según sus componentes estructurales y sus atributos funcionales y delimitado cartográficamente, según su expresión paisajística, acoge en su seno a otros sistemas naturales, de un nivel escalar inferior y forma parte integrante de una nueva entidad, perteneciente a un orden superior de magnitud (Mardones, 2006; Borja et al. 2008). Esta consideración jerarquizada del territorio es susceptible de ser representada cartográficamente, utilizando diferentes niveles de resolución (Montes et al. 1998), lo que supone una inestimable ventaja, ya que ello permite trabajar con la escala de análisis y gestión más adecuada, para cada problemática territorial (Galvis, 2007; Borja \& Montes, 2008).

Desde el punto de vista operativo, la regionalización ecológica es la principal herramienta con la que cuenta el enfoque ecosistémico para su despliegue y su puesta en valor. Se trata de un proceso consistente, en esencia, en la clasificación jerárquica de los ecosistemas presentes en un determinado territorio y en la plasmación cartográfica de los mismos, según distintos órdenes escalares; esto es, en la identificación, la caracterización y la discriminación cartográfica, a diferentes escalas espacio-temporales, de dichos ecosistemas (Borja et al. 2004; 2009). Dado que mediante los oportunos procesos de agregación o segregación de los sistemas naturales podemos identificar nuevos ecosistemas a otras escalas, la regionalización ecológica no solamente permite establecer las relaciones de dependencia existentes entre los ecosistemas de un mismo escalafón, sino que, merced a su carácter jerárquico, también lo hace respecto de los vínculos existentes entre los ecosistemas integrados en sucesivos órdenes de magnitud, lo que refuerza su carácter integral, como procedimiento analítico.

Acorde con los trabajos de Klijn $(1991 ; 1994)$ y Klijn \& De Haes (1994), en el esquema de clasificación jerárquica de ecosistemas que seguimos en el presente trabajo (Borja et al. 2004; 2008; 2009), se identifica un total de nueve órdenes escalares; de mayor a menor tendríamos: ecozona, ecodominio, ecoárea, ecoprovincia, ecoregión, ecodistrito, ecosección, ecotopo y ecoelemento (Tabla 1). Los ecosistemas integrados en cada uno de estos niveles se caracterizan, tanto desde el punto de vista estructural (principales componentes del sistema natural a cada escala o factores de control) como funcional (capacidad dinámica, de evolución y de permanencia de los ecosistemas), además de quedar agrupados según su perspectiva temporal de cambio, ya venga éste motivado por factores de carácter geológico, climático o antrópico. Una vez convenientemente jerarquizados, los factores de control (un total de 34, según la propuesta recogida en la Tabla 1), operan a modo de criterios de caracterización y de selección de los ecosistemas por cada escalafón. De ahí, la importancia de llevar a cabo una selección lo más pertinente posible de dichos factores de control, a fin de que tengan utilidad suficiente, tanto para definir y clasificar los ecosistemas de cada orden escalar como para reconocerlos y delimitarlos espacialmente (Montes et al. 1998).

Según se indicó anteriormente, la gestión ambiental basada en el enfoque ecosistémico requiere de unidades de análisis y gestión que, además de ser homogéneas en lo ecológico, también lo sean desde el punto de vista socio-económico. Este tipo de demarcaciones, a las que, siguiendo la propuesta de Borja y colaboradores para el caso de Andalucía (España), hemos denominado Unidades Ecológicas de Gestión (UEG), constituyen ámbitos territoriales, cuya homogeneidad viene definida por la integración de las tramas ecológica y socio-económica. En el caso que nos ocupa y a diferencia con el ejemplo Andaluz, donde las UEG se obtuvieron mediante la agregación de ecosistemas a escala de ecorregión en función de la homogeneidad de sus paisajes, la trama ecológica del Distrito de Cartagena de Indias equivale a la 
clasificación de sus ecosistemas a escala de ecosección, mientras que la trama socio-económica se identifica, básicamente, mediante la asignación a cada uno de los ecosistemas delimitados de unos determinados usos del territorio y una dinámica socio-económica particular.

Conceptualmente, las UEG son equiparables a la noción tradicional de los paisajes culturales, entendidos como el resultado de la acción moduladora de los humanos sobre el medio (Borja \& Montes, 2008; Borja et al. 2009) o, asimis- mo, desde la perspectiva del Cambio Global, con la expresión más actual de los socio-ecosistemas (Anderies et al. 2004). Por el contrario, su significado difiere en esencia del asignado, por ejemplo, a las Unidades Comuneras de Gobierno, sobre las que se basa la actual organización territorial por localidades de Cartagena (Alcaldía Mayor de Cartagena de Indias, 2002), cuya concepción responde, primordialmente, a criterios político-administrativos, alejándose así del enfoque ecosistémico, que preside la presente propuesta.

Tabla 1. Esquema jerárquico de clasificación de ecosistemas.

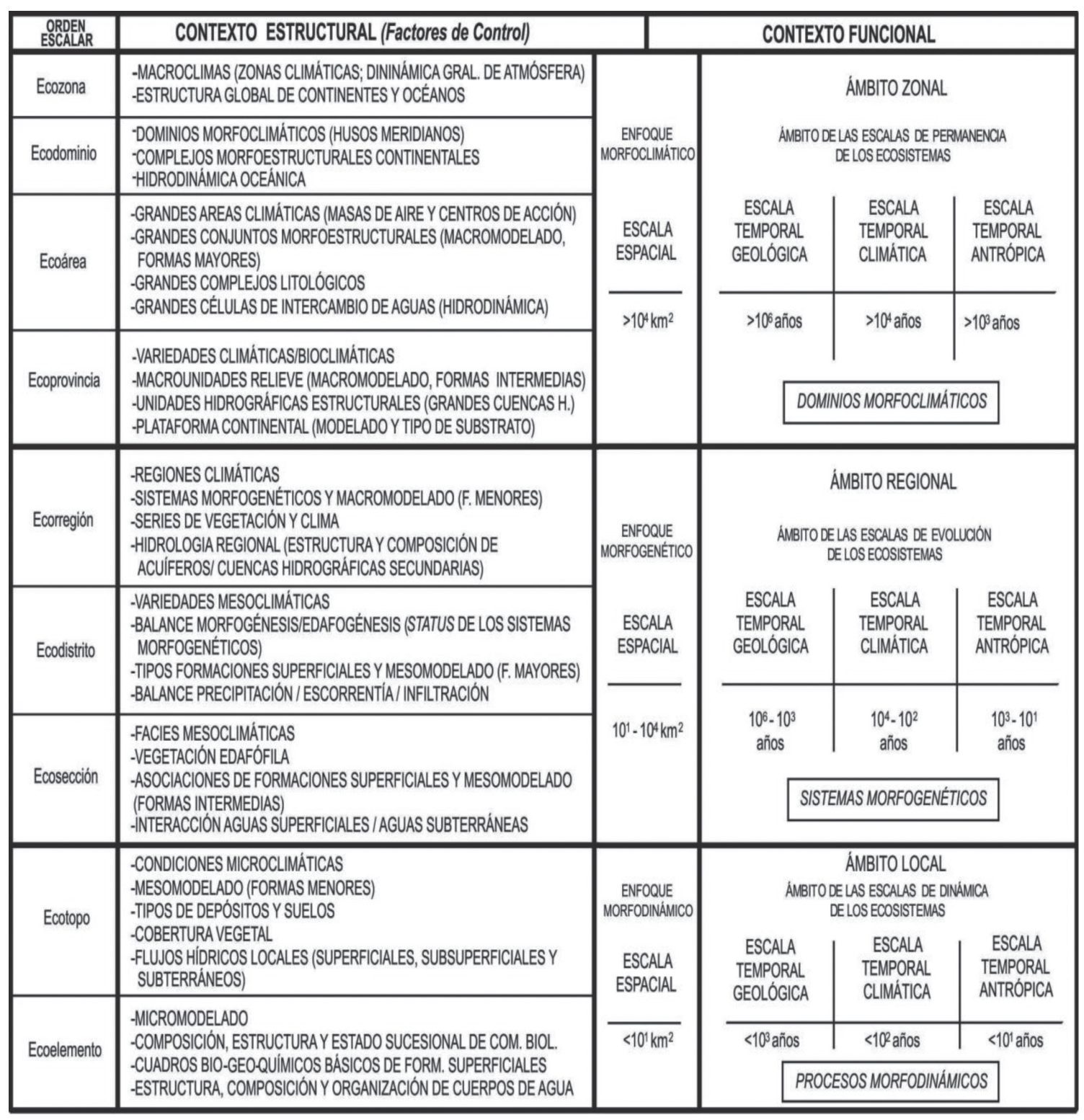

Tomado de Borja et al. 2009. 
De igual manera, la clasificación jerárquica de ecosistemas, se orienta a la búsqueda de un nivel específico de detalle, según el orden escalar seleccionado, siendo otro camino metodológico, si se compara con los diagnósticos descriptivos previos, que también abordaron el caso de Cartagena de Indias, proporcionando, en su momento, información de áreas críticas y características biofísicas, definiendo, para ello, unidades de paisaje, que enfatizaban en aspectos de macrorrelieve (Valdés, 2001).

\section{MATERIALES Y MÉTODOS}

El área de estudio: El Distrito de Cartagena de Indias esta situado a orillas del Mar Caribe, en la fachada norte de Colombia, abarca una superficie de algo más de $637 \mathrm{~km}^{2}$ (Figura
1). Su clima es de carácter tropical cálido, con temperaturas máximas medias de $31,9^{\circ} \mathrm{C}$ (agosto) y mínimas medias de $22,5^{\circ} \mathrm{C}$ (enero), que hacen una temperatura media anual de $27,2^{\circ} \mathrm{C}$, la cual, se acompañan de una humedad relativa promedio, que supera el $80 \%$ (ClOH-Cardique, 1998). Por ser una ciudad costera, se encuentra sometida al régimen de los denominados vientos Alisios. La precipitación promedio total anual es de $976 \mathrm{~mm}$ y un promedio mensual $51,4 \mathrm{~mm}$, siendo los meses de más lluvias el periodo de mayo a noviembre, con máxima precipitación en octubre y los meses de menos lluvias de diciembre a abril (CIOH, 2010).

Procedimientos y enfoque metodológico: Desde el punto de vista del procedimiento cartográfico, el material de referencia utilizado ha sido el disponible en la base on line del SIGOT

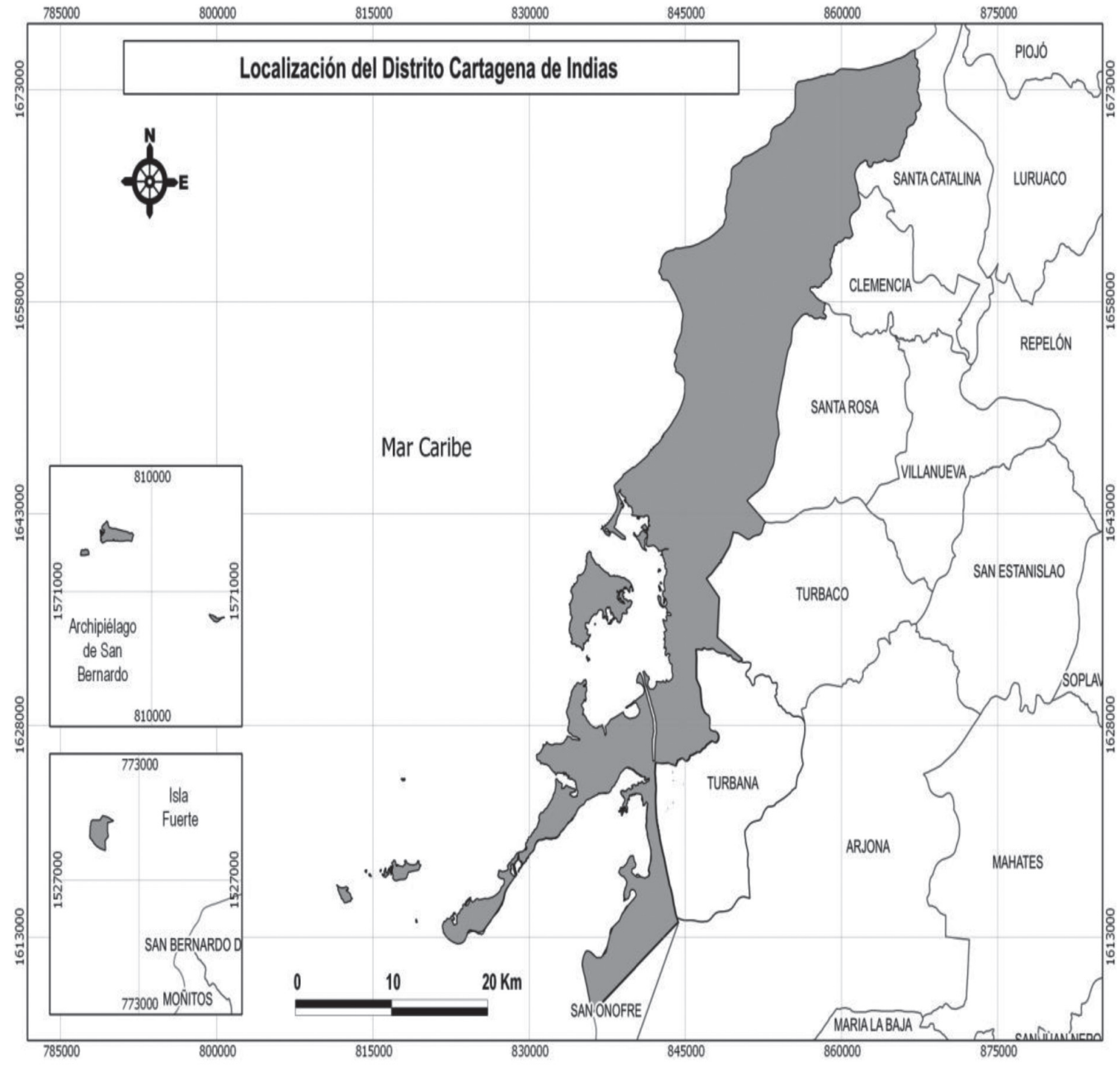

Figura 1: Localización del Distrito de Cartagena de Indias. 
(IGAC), la Corporación Ambiental Cardique y los archivos cartográficos del Grupo de Investigación CTS-Universidad de Cartagena. El procesamiento de la información cartográfica, se ha realizado mediante el uso del software de código libre Quantum GIS (QGIS), en su versión QGIS 1.8.0. Las imágenes raster con información topográfica y de elevación digital fueron obtenidas a través de ASTER Global Digital Elevation Model (ASTER GDEM). El Sistema de Referencia de Coordenadas (SRC) empleado en el manejo de todas las capas, independientemente de la condición raster o vectorial, fue el MAGNA -SIRGAS (Marco Geocéntrico Nacional de Referencia), teniendo en cuenta la Resolución n 068 de Enero 28 de 2005, mediante la cual se adoptó el MAGNASIRGAS como único datum oficial de Colombia (Diario Oficial, 2005). Finalmente, la selección de los factores de control y la identificación de Unidades Ecológicas de Gestión, realizadas a partir de la regionalización ecológica del área de estudio fue llevada a cabo acorde con el procedimiento propuesto por Borja y colaboradores (2004; 2009). El orden escalar considerado para la definición y la delimitación de los ecosistemas fue el de Ecosección (Tabla 1).

\section{RESULTADOS Y DISCUSIÓN}

La regionalización ecológica aplicada al área de Cartagena de Indias ha permitido demarcar 14 ecosistemas a escala de ecosección, los cuales, una vez cualificados con la información socio-económica (datos poblacionales, usos del suelo, dinámica económica), se han transformado en otras tantas Unidades Ecológicas de Gestión (Tabla 2 y Figura 2). De entrada, esta consideración de unidades homogéneas ya supone un primer criterio de gran interés para la gestión ambiental del Distrito de Cartagena y los planes de ordenamiento a nivel de cada localidad, cuya normativa obliga a considerar las homogeneidades ecológicas y socio-económicas, presentes en el territorio distrital.

La proporción superficial relativa de cada una de estas unidades homogéneas, se expresa en la figura 3 , donde se aporta información comparativa sobre sus diferentes magnitudes espaciales. Los resultados obtenidos muestran que la UEG Llanura Costera Rural de la Zona Norte ocupa la mayor proporción superficial relativa, con el 25,8\% del total del área Distrital, seguida de las UEG Colinas y Lomas Distritales $(15,4 \%)$, Territorios Insulares $(13,6 \%)$ y Planicie Rural Cartagena-Bayunca $(13,1 \%)$.

En la Llanura Costera Rural de la Zona Norte, se encuentran los asentamientos de Arroyo de las Canoas, Las Europas, Arroyo de Piedra, Punta Canoa, Manzanillo del Mar y Pontezuela, que suman un total de 2.594 habitantes, según proyección del censo DANE (2005); se constituye así en la

Tabla 2. Unidades Ecológicas de Gestión identificas en el Distrito de Cartagena.

\begin{tabular}{|c|c|c|c|c|}
\hline $\mathrm{N}^{\circ}$ & Unidad Ecológica de Gestión identificada & $\begin{array}{l}\text { Área } \\
\left(\mathrm{km}^{2}\right)\end{array}$ & Población & $\begin{array}{c}\text { Densidad Pobla- } \\
\text { cional Hab/km² }\end{array}$ \\
\hline 1 & UEG Llanura Costera Rural de la Zona Norte & 164,34 & 2.594 & 16 \\
\hline 2 & UEG Colinas y lomas Distritales & 98,62 & 190.624 & 1933 \\
\hline 3 & UEG Plano ondulado de Canalete & 4,38 & - & - \\
\hline 4 & UEG Planicie Rural Cartagena-Bayunca & 83,52 & 7.410 & 89 \\
\hline 5 & UEG Humedal Ciénaga de la Virgen & 22,84 & - & - \\
\hline 6 & UEG Bosque de Manglar de la Ciénaga & 7,87 & - & - \\
\hline 7 & UEG Frente Litoral Boquilla-Crespo & 4,06 & 22.456 & 5531 \\
\hline 8 & $\begin{array}{l}\text { UEG Planicie de los Caños y Lagunas Interi- } \\
\text { ores }\end{array}$ & 5,80 & 96.127 & 16574 \\
\hline 9 & UEG Cerro de la Popa & 2,98 & 56.119 & 18832 \\
\hline 10 & UEG Planicie Urbana de Cartagena & 22,35 & 434.749 & 19452 \\
\hline 11 & UEG Territorios Insulares & 86,93 & 10.695 & 123 \\
\hline 12 & UEG Planicie Litoral de la Bahía de Cartagena & 52,95 & 132.774 & 2508 \\
\hline 13 & UEG Planicie Litoral de la Bahía de Barbacoas & 61,65 & 3.511 & 57 \\
\hline 14 & UEG Planicie del Canal del Dique & 19,50 & 10.044 & 515 \\
\hline
\end{tabular}

Elaboración propia. Base Censal DANE 2005 proyección 2012. 


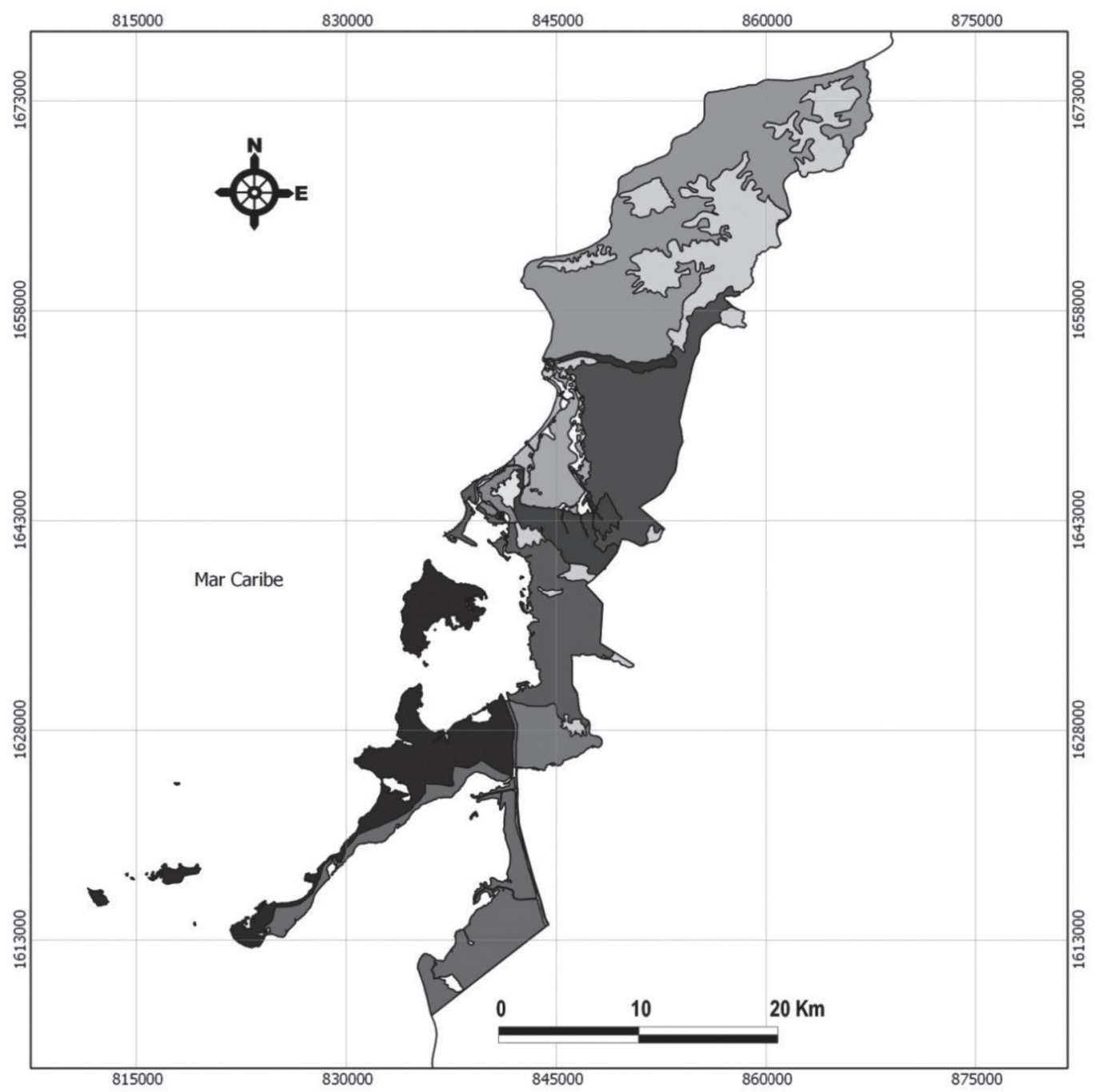

\section{Simbología}

Unidades Ecológicas de Gestión

$\square$ Bosque de Manglar de la Ciénaga

Cerro de la Popa

Colinas y Lomas Distritales

Frente Litoral Boquilla-Crespo

Humedal Cienaga de la Virgen

Llanura Costera Rural de la Zona Norte

Planicie de los Caños y Lagunas Interiores

Planicie del Canal del Dique

Planicie Litoral de la Bahia de Barbacoas

Planicie Litoral de la Bahia de Cartagena

Planicie Rural Cartagena-Bayunca

Planicie Urbana de Cartagena

Plano Ondulado de Canalete

Territorios Insulares

845000

\section{Mapa de Unidades Ecológicas de Gestión (UEG) del Distrito Cartagena de Indias}

Sistema de Referencia de Coordenadas: MAGNA- SIRGAS

Procesamiento información: Software QGIS 1.8.0

Base Cartografica: SIGOT-IGAC; Cardique; Grupo CTS

Universidad de Cartagena

Equipo SIG: E. Torregroza-Fuentes y A. Gómez-Juan

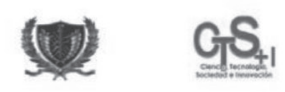

Figura 2. Mapa de las Unidades Ecológicas de Gestión del Distrito de Cartagena de Indias. 


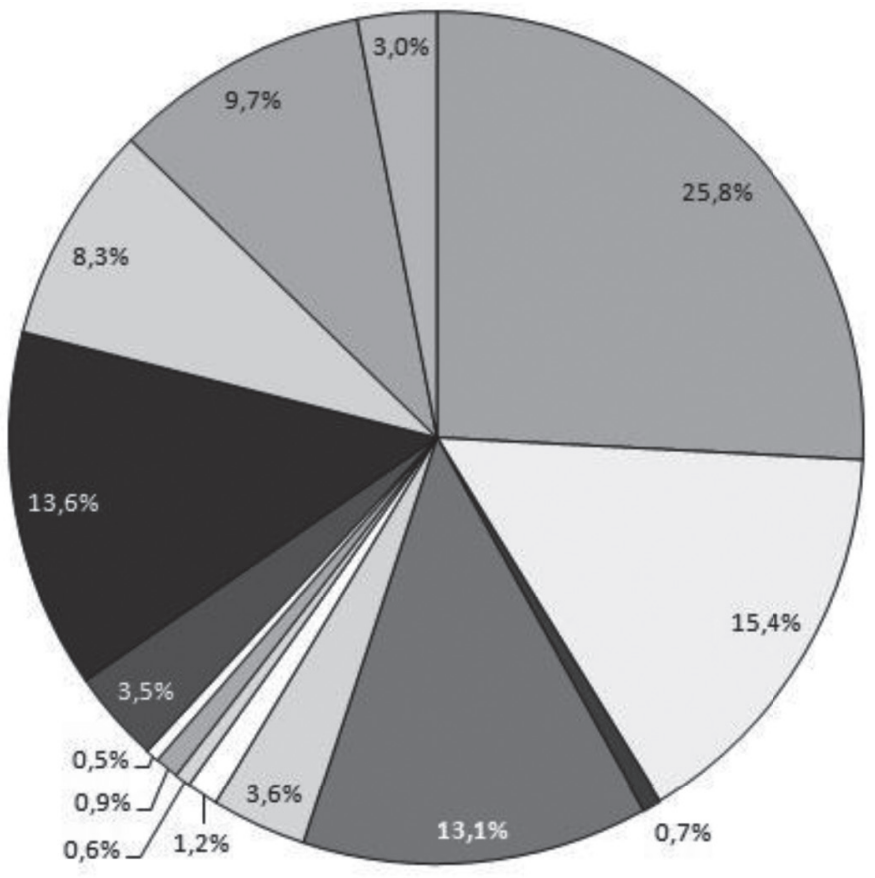

\author{
口 Llanura Costera Rural de la Zona Norte \\ $\square$ Colinas y lomas Distritales \\ - Plano ondulado de Canalete \\ $\square$ Planicie Rural Cartagena-Bayunca \\ $\square$ Humedal Ciénaga de la Virgen \\ $\square$ Bosque de Manglar de la Ciénaga \\ $\square$ Frente Litoral Boquilla-Crespo \\ $\square$ Planicie de los Caños y Lagunas Interiores \\ $\square$ Cerro de la Popa \\ - Planicie Urbana de Cartagena \\ - Territorios Insulares \\ $\square$ Planicie Litoral de la Bahía de Cartagena \\ 口Planicie Litoral de la Bahía de Barbacoas \\ $\square$ Planicie del Canal del Dique
}

Figura 3. Distribución superficial proporcional de las UEG, en el Distrito de Cartagena de Indias.

UEG de menor densidad poblacional, con sólo $16 \mathrm{hab} / \mathrm{km}^{2}$. Su población es mayoritariamente afrodescendiente y se dedican a labores de pesca y de agricultura. El potencial turístico de sus playas, unido a las tendencias urbanísticas de la elite social, constituyen, de hecho, variables a tener muy en cuenta por los tomadores de decisiones, a nivel de implementación políticas públicas, dado que las poblaciones originales presentan muchas necesidades básicas insatisfechas y los medios litorales son, en general, altamente sensibles al incremento de la carga poblacional y al impacto del "urbanismo de hormigón".

La Unidad de Colinas y Lomas Distritales ocupa $98,62 \mathrm{~km}^{2}$, el equivalente a un 15,4\% del área de estudio y acoge a unos 190.624 habitantes, que supone una densidad poblacional de $1.933 \mathrm{hab} / \mathrm{km}^{2}$. En esta UEG destaca el ámbito denominado Cerro de Púa, localmente llamado ahora por los pobladores de Arroyo de Piedra, como "Cerro de las Torres", por tener instaladas, en su parte más alta, antenas del servicio de telefonía móvil. Esta elevación ocupa un área de $6,27 \mathrm{~km}^{2}$, siendo algo más del doble de la superficie que ocupa el Cerro de la Popa; sin embargo, este último presenta un interés mayor por parte de ciudadanos y tomadores de decisiones, en gran medida, gracias al hecho de estar inmerso en el casco urbano de Cartagena, así como debido al sentido religioso del sitio, ya que en su cima acoge la iglesia dedicada a la Virgen de la Candelaria. Dentro de esta unidad ecológica también destaca un área de lomerío, que constituye la divisoria de aguas entre las cuencas de la Ciénaga de la Virgen y la Bahía de Cartagena.

La Planicie Rural Cartagena-Bayunca forma parte realmente de una llanura mayor (Cartagena-Santa Rosa), localizada a levante de la Ciénaga de la Virgen, a cuyo borde oriental entregan sus aguas los arroyos Mesa, Tabacal, Hormiga y Chiricoco. Su delimitación dentro del contexto distrital viene dada por el límite político-administrativo correspondiente a la carretera La Cordialidad (Ruta 90, Nacional), que marca la frontera del municipio de Santa Rosa de Lima con el Distrito de Cartagena de Indias, concretamente, con el área correspondiente al corregimiento de Bayunca. Además del asentamiento de Bayunca, en esta UEG también se encuentran otros poblados ubicados al oeste de la Carretera La Cordialidad, situación que empieza a marcar el cambio en el uso del suelo que caracterizaba este territorio; no obstante, esta unidad no ha perdido en absoluto su condición prioritariamente rural, lo que mantiene su densidad poblacional en uno de los niveles más bajos área de estudio $\left(89 \mathrm{hab} / \mathrm{km}^{2}\right)$. Valoradas sus características desde los principios de la Evaluación de los Ecosistemas del Milenio, efectivamente, esta UEG quedaría tipificada como perteneciente a la categoría de los agroecosistemas, en la medida en que su paisaje ha 
sido gestionado y modificado por sus habitantes fundamentalmente, para la obtención de alimentos y otros materiales de origen biótico (Evaluación de Ecosistemas del MilenioEspaña, 2013).

Las UEG de menor proporción relativa, pero no por ello de menor importancia, a nivel de dinámica territorial, son las del Cerro de la Popa $(0,5 \%)$ y el Frente Litoral Boquilla-Crespo $(0,6 \%)$. Estas unidades, junto con las correspondientes a la Ciénaga de la Virgen, el Bosque de Manglar y el Plano ondulado de Canalete son los principales componentes del paisaje de la Cuenca de la Ciénaga de la Virgen. De forma particular, los resultados obtenidos para estos espacios vienen a confirmar que se trata de unidades sometidas a una alta presión antrópica. El proceso de "colonización", por ejemplo, del Cerro de la Popa, cuya densidad de población alcanza los $18.832 \mathrm{hab} / \mathrm{km}^{2}$, se inició en torno a 1961 (Barboza \& Carvajal, 2007), viéndose acompañado de una intensa deforestación, así como de la subsiguiente remoción de suelos, hasta verse comprometida la estabilidad de sus laderas. La UEG Frente Litoral Boquilla-Crespo, por su parte, se caracteriza por presentar una interesante flora Psammofila, en la que destacan el cocotero (Coccus nucifera) y la uvita de playa (Coccoloba uvifera). En los últimos años, esta unidad se ha visto afectada por una fuerte presión provocada por el turismo de "sol y playa" y por un creciente proceso de urbanización, fruto de la litoralización potenciada por el negocio de finca raíz. Esta transformación no solamente afecta a la vegetación nativa sino también y, de manera muy especial, a la población de pescadores afrodescendientes, quienes habitaban, originalmente, en el Corregimiento de La Boquilla. También hay que indicar que 17,46 ha de los $4,06 \mathrm{~km}^{2}$ que ocupa la unidad están destinadas a las infraestructuras del Aeropuerto Internacional Rafael Núñez.

Por su parte, el Humedal Ciénaga de la Virgen y el Bosque de Manglar de la Ciénaga, con el 3,6\% y el 1,2\%, respectivamente, de la superficie del Distrito, constituyen los ecosistemas que aportan uno de los flujos de servicios ecosistémicos más importante para el bienestar de los habitantes de la zona; sin embargo, aspectos derivados de la presión antrópica, sobre todo los que atañen a la zona sur del humedal, el que linda con la Planicie Urbana de Cartagena, constituyen factores de cambio que están amenazando seriamente la integridad ecológica de estos ecosistemas y con ello, la posibilidad que sigan aportando beneficios a la comunidad. En la actualidad, tanto los 22,84 $\mathrm{km}^{2}$ de la lámina de agua del humedal como los $7,87 \mathrm{~km}^{2}$ del bosque de mangle forman parte del Parque Distrital Ciénaga de la Virgen, que cuenta, al menos en teoría, con un plan distrital orientado a su preservación (Alcaldía Mayor de Cartagena, 2006); no obstante, la tala indiscriminada de mangle y el posterior relleno del humedal son prácticas que no encuentran freno, muy a pesar de la abundante normativa existente al respecto. Esta "manglefobia" nos es más que una de las secuelas de la vorágine en la que se ve envuelto este territorio, cuya apetencia por la tierra y el afán por el lucro derivado del negocio inmobiliario de las élites locales y foráneas, solamente se ve superada por la pobreza de quienes allí carecen de espacios para una vivienda digna.

La UEG Planicie de los Caños y Lagunas Interiores, con un $0,9 \%$ de la extensión distrital), corresponde al área dinamizada por el sistema de drenajes y de humedales que conecta la Ciénaga de la Virgen con la Bahía de Cartagena. En ella, destaca el caño Juan Angola, que enlaza directamente la aludida ciénaga con las lagunas de San Lázaro, Chambacú y el Cabrero, conservando en sus márgenes bosques relictos de manglar, muy afectados por el avance urbanístico y la apertura de nuevos tramos de carreteras. La incidencia antrópica en la Planicie y evidentemente en los cuerpos de agua del sistema de caños y de lagunas, con alrededor de 16.574hab/ $\mathrm{km}^{2}$, hace de esta Unidad una de las más afectadas por la acción humana, de ahí que requiera con urgencia de un plan integral de restauración.

La UEG Planicie Urbana de Cartagena, por su parte, abarca el 3,5\% de la extensión Distrital y es un claro ejemplo de lo que, a nivel de Ecosistemas del Milenio, se ha dado en llamar un "ecosistema urbano", siendo el área de mayor concentración humana $\left(19.452 \mathrm{hab} / \mathrm{km}^{2}\right)$. También es importante señalar que esta Unidad Ecológica de Gestión está surcada por los drenajes de los arroyos Limón o Tomatal y Matute, así como por el desagüe urbano de Cartagena, cuya red tributa hacia la Ciénaga de la Virgen y se ve sometida a un intenso poblamiento descontrolado en sus márgenes. Esta dinámica social de "tugurización" ha provocado, especialmente en la zona cercana a la Ciénaga, un proceso de "creación social del riesgo" por fenómenos de inundación durante la época de lluvias, muy similar al reportado por Beraud et al. (2007), en regiones de México, con vulnerabilidad de la población.

La UEG Planicie Litoral de la Bahía de Cartagena representa el 8,3\% del territorio distrital, contiene la denominada zona histórica, muy reconocida por la ciudad colonial, así como el área turística tradicional de los Barrios de Bocagrande, Castillo Grande y la franja litoral del Barrio Manga. También abarca toda la zona de tierra firme asociada con la Bahía de Cartagena, incluyendo el área de puerto y la denominada zona industrial de Mamonal. Se podría decir que, como ciudad, Cartagena palpita, se mira y se piensa priorizando esta UEG, donde se concentran los centros de poder, de desarrollo turístico y de actividad portuaria y de logística de la zona. A esta dinámica, se une la actual apetencia por las UEG Territorios Insulares y las ya comentadas del Frente Litoral Boquilla-Crespo y la Llanura Costera Rural Norte. Situación que se evidencia al examinar la ubicación espacial de la pobreza en el territorio distrital, tal como lo describen los trabajos sobre economía regional de Pérez \& Salazar (2007). La existencia 
de casi 60 muelles con una actividad estimada de unos 500 buques al mes haciendo uso de las infraestructuras portuarias (Informe Geo-Cartagena, 2009), podría servir de indicador del elevado dinamismo de esta unidad ecológica.

En cuanto a la UEG Planicie Litoral de la Bahía de Barbacoas, con un 9,7\% del espacio distrital, está muy influenciada por la dinámica territorial de la Bahía que lleva su nombre, caracterizada por un alto potencial turístico gracias a la inclusión dentro de la misma de la Isla de Barú. En contraposición, esta Unidad también acoge los asentamientos de Leticia, Recreo y Santa Ana, en los que predomina la población afrodescendiente.

La UEG Llanura del Canal del Dique es, con un 3\% del territorio de Cartagena, un área cuya singularidad deriva, principalmente, de la presencia del canal de comunicación entre el río Magdalena con la Bahía de Cartagena. Este lecho artificial tiene sus orígenes en la época colonial y responde al afán de lograr una conexión fluvial de Cartagena con el interior del país. En la actualidad y tras una serie de "rectificaciones" que eliminaron las grandes curvas en su trazado inicial, es una importante vía de entrada de sedimentos a la bahía. Esta Unidad territorial incluye asentamientos, como el Corregimiento de Pasacaballos, de población afrodescendiente vinculados a la pesca artesanal, en unas condiciones muy similares a las que se dan en el Corregimiento de la Boquilla, del que se diferencia por su menor densidad poblacional (515hab $\left./ \mathrm{km}^{2}\right)$. Se prevé que esta situación puede sufrir variaciones en un futuro inmediato, en la línea de la tendencia urbanística ya planteada para la UEG Frente Litoral Boquilla-Crespo.

Por último, la UEG Territorios Insulares representa, en su conjunto, alrededor del 13,6\% de la circunscripción del Distrito de Cartagena, destacando en ella la presencia del Parque Nacional Natural Corales del Rosario y San Bernardo que, por su condición marítimo-terrestre incluye los territorios costeros, situados hasta los $50 \mathrm{~m}$ de profundidad. Este sistema de islas tiene implicaciones ecológicas de altísimo interés derivadas de la presencia de arrecifes de coral y de especies asociadas a ellos, como al manglar.

Conclusiones: Se evidenció que la regionalización ecológica, herramienta básica de la aproximación ecosistémica, constituye una metodología viable para la identificación de unidades homogéneas desde el punto de vista de sus tramas ecológica y humana (Unidades Ecológicas de Gestión), las cuales, son de gran utilidad en el diagnóstico ambiental. Aplicado al Distrito de Cartagena de Indias, dicho procedimiento de regionalización ecológica ha permitido caracterizar y delimitar catorce (14) UEG, que han sido discriminadas a partir de la identificación de ecosistemas a la escala de ecosección y de la asignación de los usos del territorio y los atributos socio-económicos de cada uno de ellos. Merced a dicho tratamiento podemos afirmar que en el Distrito de Cartagena de Indias ha venido desarrollándose un proceso cada vez más acentuado de litoralización, que está afectando gravemente a la integridad ecológica de la totalidad de los ecosistemas litorales. Esta situación ha venido motivada, como en otras partes del mundo, por el incremento de las actividades derivadas del turismo de sol y playa, las apetencias sociales por los emplazamientos costeros. A este respecto, destaca la UEG identificada como el Frente Litoral Boquilla-Crespo y la UEG Planicie Litoral de la Bahía de Cartagena, cuyos elevados requerimientos de áreas industriales y espacios para grandes infraestructuras de puertos, relacionados, incluso con la exportación minera carbonífera, sugieren llevar a cabo estudios de mayor detalle, que permitan sopesar en su real dimensión los conflictos de intereses y el balance de ventajas y desventajas (trade off), que trae consigo la situación actual. Queda igualmente reafirmado que la sostenibilidad solamente se puede garantizar con capacidad política, desde un punto de vista integral y de justicia social, alejada de los tradicionales enfoques sectoriales.

Agradecimientos: A la Universidad de Cartagena, por el apoyo financiero dado a esta investigación, en el marco de la IV Convocatoria para proyectos de Semilleros de Investigación. Igualmente, al doctor Eduardo Caro, de Arizona State University (USA), por su apoyo en la revisión del summary. Conflicto de intereses: El artículo fue preparado y revisado con la participación de todos los autores, quienes declaramos que no existe ningún conflicto de intereses que ponga en riesgo la validez de los resultados presentados.

\section{BIBLIOGRAFÍA}

1. ANDERIES, J.M.; JANSSEN, M.A.; OSTROM, E. 2004. A framework to analyze the robustness of socialecological systems from an institutional perspective, Ecology and Society 9(1):18. Disponible desde internet en: http://www.ecologyandsociety.org/vol9/iss1/ art18/ (con acceso el 23/07/2013).

2. ALCALDÍA MAYOR DE CARTAGENA DE INDIAS. 2002. Acuerdo No 26 del 26 de Diciembre de 2002 por el cual se señalan las localidades del territorio del Distrito turístico y cultural de Cartagena de indias, su denominación, límites y atribuciones administrativas y se dictan otras disposiciones para su organización y funcionamiento. $7 p$.

3. ALCALDÍA MAYOR DE CARTAGENA DE INDIAS. 2006. Decreto $N^{\circ} 063$ de 2006 emanado por la Alcaldía Mayor de Cartagena de Indias el 23 de Enero de 2006. Por el cual se establece el Macroproyecto Parque Distrital Ciénaga de la Virgen. 29p. 
4. BARBOZA, G.; CARVAJAL, J. 2007. Caracterización geotécnica y análisis de la susceptibilidad a los fenómenos geológicos en el casco urbano de Cartagena. Rev. Ciencias e Ingeniería al Día. 3(1):21-34.

5. BERAUD, J.L.; COVANTES, C.; BERAUD, I.P. 2007. Construcción social de las condiciones de riesgo en Mazatlán, Sinaloa. Revista Electrónica Zacatecana sobre Población y Sociedad. Año 7, número 31, septiembre-diciembre, Universidad Autónoma de Zacatecas, México, 18p. Disponible desde internet en: http://sociales.uaz.edu.mx/c/document_library/get_file?uuid=ac992580-85bd-431d-bb4e7363e22ce69f\&groupld=12606 (con acceso el 23/07/2013).

6. BORJA, F; MONTES, C. 2008. La gestión ecosistémica como herramienta territorial para la toma de decisiones. Ecorregiones e integración funcional de carreteras y espacios naturales protegidos en Andalucía. Santander España. Disponible desde internet en:http://www.aecarretera.com/Ponencia\%20F\%20 Borja\%20AEC\%20Santaber\%2008\%20def.pdf (con acceso 23/07/20013).

7. BORJA, F.; ROMÁN, J.M.; MONTES, C.; MOREIRA, J.M.; RODRÍGUEZ, J. 2004. Regionalización Ecológica de Andalucía y Unidades Ecológicas de Gestión en el marco del Plan Director de la RENPA. Consejería de Medio Ambiente. Junta de Andalucía. Sevilla. 85p.

8. BORJA, F.; ROMÁN, J.M.; BORJA, C. 2008. Regionalización ecológica de la vega y marisma del Guadiamar. Aproximación a la trama biofísica del Corredor Verde del Guadiamar. En: La Restauración ecológica del Río Guadiamar y el Proyecto del Corredor Verde. Cons. de Medio Ambiente. Junta de Andalucía. Sevilla. p.91-100.

9. BORJA, F; MONTES, C.; ROMÁN, J.M. 2009. Regionalización Ecológica y Red de Espacios Natrales Protegidos de Andalucía. En: Proyecto Andalucía. Naturaleza. Vol. 31 (Ecología IV). Publicaciones Comunitarias. Grupo Hércules. Sevilla. p.119-154.

10. CIOH - CARDIQUE. 1998. Caracterización y diagnóstico integral de la zona comprendida entre Galerazamba y bahía Barbacoas. Tomo II Caracterización. Centro de Investigaciones Oceanográficas e Hidrográficas. Cartagena - Colombia. p 412.

11. CIOH. 2010. Climatología de los principales puertos del Caribe Colombiano- Cartagena de Indias D. T. y C. 11p. Disponible desde internet en: http://www.cioh.
org.co/meteorologia/Climatologia/ Climatologia\%20 Cartagena.pdf (con acceso el 27/12/2013).

12. DANE. 2005. Censo General 2005- Nivel Nacional. Departamento Administrativo Nacional de Estadística. 498p.

13. DIARIO OFICIAL. 2005. Resolución 068 de Enero 28 de 2005, mediante la cual se adopta MAGNA-SIRGAS como el único datum oficial de Colombia. República de Colombia. $2 p$.

14. EVALUACIÓN DE LOS ECOSISTEMAS DEL MILENIO ESPAÑA - EME. 2013. Guía del Profesorado. www. ecomilenio.es. Disponible desde internet en http:// www.ecomilenio.es/wp-content/uploads/2013/07/ Gu\%C3\%Ada-profesarado (con acceso el 23/07/2013).

15. FOLKE, C. 2006. The Economic Perspective: Conservation against Development versus Conservation for Development. Conserv. Biol. 20:686-688.

16. GALVIS, M.F. 2007. La generalización en cartografía básica y temática. Rev. Ingeniería y Tecnología. 9:207239.

17. GRUMBINE, R.E. 1994. What is ecosystem management? Conserv. Biol. 8(1):27-38.

18. INFORME GEO-CARTAGENA. 2009. Perspectivas del Medio Ambiente Urbana. Programa de las Naciones Unidas para el Medio Ambiente (PNUMA), Alcaldía de Cartagena de Indias, Establecimiento Público Ambiental de Cartagena-EPA Cartagena y el Observatorio del Caribe Colombiano.172p.

19. JIANGUO, L.; DIETZ, T.; CARPENTER, S.; ALBERTI, M.; FOLKE, C.; MORAN, E.; PELL, A.; DEADMAN, P.; KRATZ, T.; LUBCHENCO, J.; OSTROM, E.; OUYANG, Z.; PROVENCHER, W.; REDMAN, C.; SCHNEIDER, S.; TAYLOR. W. 2007. Complexity of coupled Human and Natural Systems. Science. 317:1513.

20. KLIJN, F. 1991. Hierarchical classification of ecosystems: a tool for susceptibility analysis and quality evaluation for environmental policy. In: Ravera, O. (ed.) Terrestrial and aquatic ecosystems. Perturbation and recovery. Ellis Horwood, Chichester. p.80-89.

21. KLIJN, F. 1994. Spatially nested ecosystems - guidelines for classification from a hierarchical perspective. In: Klijn, F. (ed.). Ecosystem Classification for Environ- 
mental Management: Dordrecht, The Netherlands, Kluwer Academic Publishers. p.85-116.

22. KLIJN, F.; DE HAES, H.U. 1994. A hierarchical approach to ecosystems and its implications for ecological land classification. Landscape Ecol. 9(2):89-104.

23. MARDONES, G. 2006. Clasificación jerárquica y cartografía de ecosistemas en la zona andina de la Región del Biobío, Chile. Rev. Geografía Norte Grande. 35:59-75.

24. MILLENNIUM ECOSYSTEM ASSESSMENT. 2003. Ecosystems and human well-being: A framework for the assessment. Island Press, Washington D.C., USA, 212p.

25. MITCHELL, B. 1999. La gestión de los recursos naturales y del medio ambiente. Madrid. Ediciones MundiPrensa. 290p.

26. MONTES, C.; BORJA, F.; BRAVO, M.; MOREIRA, J. 1998. Reconocimiento biofísico de espacios naturales protegidos. Doñana: Una aproximación ecosistémica. Junta de Andalucía. Consejería de Medio Ambiente. 309p.

27. MORIN, E. 2001. Los siete saberes necesarios para la educación del futuro. Editorial Mesa Redonda. Organización de las Naciones Unidas para la Educación, la Ciencia y la Cultura. Cooperativa Editorial Magisterio. Bogotá. Colombia. 67p.

28. NICOLESCU, B. 1999. La transdisciplinariedad: Una nueva visión del mundo. En La Transdiscipglinariedad. Ed. du Rocher-Monaco.Collection "Transdisciplinarité". Disponible desde internet en: http:// nicol.club.fr/ciret/espagnol/visiones.htm (con acceso 18/02/2013)

29. PÉREZ, G.J.; SALAZAR, I. 2007. La pobreza en Cartagena: Un análisis por barrios. Documentos de trabajo sobre economía regional. Banco de la República. Centro de Estudios Económicos Regionales (CEER)Cartagena. Disponible desde internet en: http://www. banrep.gov.co/publicaciones/pub_ec_reg4.htm (con acceso el 21/07/2013).

30. SALA, O.; CHAPIN, S.; ARMESTO, J.; BERLOW, E.; BLOOMFIELD, J.; DIRZO, R.; HUBER-SANWALD, E.; HUENNEKE, L.; JACKSON, R.; KINZIG, A.; LEEMANS, R.; LODGE, D.; MOONEY, H.; OESTERHELD, M.; LEROY POFF, N.; SYKES, M.; WALKER, B.; WALKER, M.; WALL, D. 2000. Global biodiversity scenarios for the year 2100. Science. 287:17701774.

31. UNEP. 2006. Ecosystem Bases Management. Markers for assessing progress. UNEP, GPA. 49p.

32. VALDÉS, C. 2001. Unidades de Paisaje. En "Lineamientos para el ordenamiento territorial del Distrito Turístico y Cultural de Cartagena De Indias". Serie Investigación 3. Editor: Ernesto Puertas. IDEADE, Pontificia U. Javeriana. Bogotá, D.C. Colombia. Ed.Fundación Cultural Javeriana de Artes Gráficas Javegraf. p.139160

33. YAFFEE, S.L. 1999. Three faces of ecosystem management. Conserv. Biol. 13(4):713-725.

Recibido: Julio 29 de 2013

Aceptado: Febrero 18 de 2014

Como citar:

Torregroza, E.; Hernández, M.; Barraza, D.; Gómez, A.; Francisco Borja, F. 2014. Unidades ecológicas para una gestión ecosistémica en el distrito Cartagena de Indias (Colombia). Rev. U.D.C.A Act. \& Div. Cient. 17(1): 205-215. 
